\title{
Las tecnologías de Linked Data y sus aplicaciones en el gobierno electrónico
}

\author{
Linked Data technologies and their application to e-government
}

\author{
María Asunción Hallo (1), María Mercedes MARTínez-GonzÁlez, Pablo de la FuENTE Re- \\ DONDO (2)
}

(1) Departamento de Informática y Ciencias de Computación, Escuela Politécnica Nacional, Quito (Ecuador), maria.hallo@epn.edu.ec (2) Departamento de Informática, Universidad de Valladolid (España), \{mercedes|pfuente@uva.es\}

\begin{abstract}
Resumen
Los movimientos de acceso abierto impulsan a los organismos principalmente estatales a publicar sus datos lo más pronto posible. Las tecnologías de Linked Data ayudan en esta dirección. En este artículo se presentan conceptos y herramientas que pueden ser útiles para implementar proyectos de Linked Data, así como también algunas aplicaciones al gobierno electrónico. Nuestro objetivo es proporcionar una visión general de esta nueva área y su potencial uso, particularmente en el ámbito del gobierno electrónico. Para una implementación a gran escala es necesario desarrollar metodologías para generar y publicar Linked Data en la Web, desarrollar algoritmos para interconectar datos automáticamente, asegurar la pervivencia de los enlaces, desarrollar estándares para registrar información de las fuentes de datos, asegurar la privacidad, seguridad y preservación de los datos generados, crear herramientas para facilitar la búsqueda y despliegue de Linked Data.
\end{abstract}

Palabras clave: Administración electrónica. Web semántica. Linked Data. Gobierno electrónico.

\section{Introducción}

Actualmente hay un buen desarrollo de nuevas técnicas que tienen un gran potencial para publicar y consumir datos enlazados (Linked Data) en la Web semántica (Heath et ál., 2011). En el Organismo Internacional de estandarización de tecnologías Web W3C, se han creado grupos especializados en varias tecnologías como RDF, SPARQL, OWL para coordinar su desarrollo de estas nuevas tecnologías (Hendler, 2009). La adopción de las mejores prácticas de Linked Data ha contribuido a que se extienda el espacio global de datos conectando datos de diversos dominios. Por otra parte, el gobierno electrónico se ha impulsado en varios países. El gobierno electrónico es sinónimo de integración de servicios, procesos, sistemas, datos y aplicaciones necesarias para lograr un gobierno centrado en los ciudadanos (Codagnone et ál., 2007). El establecer un gobierno electrónico efectivo requiere apertura, transparencia, colaboración y habilidades para tomar ventaja de las

\begin{abstract}
The Open Access Initiative primary encourages state agencies to publish their data as soon as possible. Linked Data Technologies help in this direction. This article introduces concepts and tools that can help to implement projects of Linked Data, as well as some applications in e-Government. Our goal is to provide an overview of this new area and its potential use in the e-Gov field particularly. To achieve a successful implementation at a large scale, some prerrequisites must be ensured: developing methodologies to generate and publish Linked Data faithfully and economically, ensuring the persistence of links and sources, developing standards on recording metadata from this sources, ensuring data privacy, security and preservation, and developint tools to effectively and efficiently retrieve, display and navigate Linked Data.
\end{abstract}

Keywords: e-Administration. Semantic Web. Linked Data. E-Government.

capacidades de la Web (Alonso et ál., 2009). Adicionalmente, la administración electrónica, es la utilización de las Tecnologías de la Información y Comunicación como soporte del gobierno electrónico (COM, 2003).

Algunos gobiernos como Estados Unidos, Inglaterra, Francia, España, Ecuador, Argentina, incluyen en su legislación la obligación de la administración pública de transformarse en una administración electrónica en beneficio de los ciudadanos y desarrollan planes nacionales de administración electrónica (Ley 11/2007 de acceso electrónico de los ciudadanos a los Servicios Públicos, 2007; CONATEL, 2003; Montarcé, 2005; Albornoz, 2007). En el caso de España se publica en el 2010, mediante el Real Decreto 4/2010, el Esquema Nacional de Interoperabilidad que comprende un conjunto de criterios y recomendaciones que deberán ser tomados en cuenta por las administraciones públicas para la toma de decisiones tecnológicas que garanticen la interoperabilidad. En este decreto 
se menciona como una de las dimensiones la interoperabilidad semántica que requiere el establecimiento y uso de modelos de datos de naturaleza horizontal o sectorial y el establecimiento del Centro de Interoperabilidad Semántica (Amutio, 2010).

Sin embargo, debe tenerse en cuenta que generalmente en las administraciones públicas las aplicaciones son desarrolladas para cubrir servicios de unidades administrativas jerárquicas, mientras los ciudadanos tienen necesidades horizontales de acceso a servicios de múltiples agencias (Madrid, 2008). Las tecnologías de Linked Data ofrecen una posibilidad para satisfacer los requerimientos de integración horizontal y búsquedas semánticas de aplicaciones de administración electrónica, que algunos países ya empiezan a utilizar (Sheridan et ál., 2010).

El objetivo de este artículo es ofrecer una visión de las tecnologías de Linked Data, los problemas y potencialidades de aplicación a la administración electrónica. Se describen conceptos y herramientas disponibles para publicación y consumo de datos, así como las principales aplicaciones disponibles actualmente en este ámbito. Se discuten las limitaciones en implementación a gran escala y necesidades de desarrollos para facilitar su aplicabilidad.

\section{Breve historia de Linked Data}

Linked Data es un término propuesto por Tim Berners-Lee (2006) y es usado para describir un conjunto de prácticas recomendadas para publicar, compartir y conectar piezas de datos, información y conocimiento en la Web semántica usando identificadores URIs (Uniform Resource Identifiers) y RDF (Resource Description Famework) para describir los recursos (Hartig et ál., 2010).

Se pueden distinguir varios períodos en la evolución de Linked Data. Entre los años 1990 y 2000 se realizaron varios desarrollos de software para introducir representación de conocimiento principalmente en la Web de documentos. En 1999 se liberó la primera versión de RDF. La idea fue desarrollar una capa RDF sobre XML con el fin de poder usar las tecnologías como XML y XSLT. Entre 2000 y 2006 se estabilizó RDF, se añadió una semántica formal y se liberó OWL (Hausenblas, 2009). En este período existe escepticismo en que se pueda llegar a compartir datos, lo cual es actualmente superado en varios gobiernos gracias al movimiento Linked Open Data que impulsa el fácil acceso de ciudadanos y empresas a los datos públicos que recogen las Administraciones Públicas.

En el año 2006, Tim Berners-Lee escribió una nota de diseño (Berners-Lee, 2006), proponiendo soluciones a los problemas que impedían el enlace de los datos, mediante la aplicación de los principios de Linked Data que son los siguientes:

- Use URIs como nombres de recursos.

- Use HTTP URIs de manera que la gente pueda buscar esos nombres.

- Cuando alguien busque un URI, debería encontrar información útil usando los estándares RDF o SPARQL

- Incluya enlaces a otros URIs de manera que la gente pueda encontrar más recursos relacionados.

Lo anterior implica usar el modelo de datos RDF para publicar datos estructurados en la web y usar enlaces RDF para enlazar los datos de diferentes fuentes. Estos principios permitieron impulsar el desarrollo de varios proyectos de Linked Data (Heath et ál., 2011).

Por otra parte, Tim Berners-Lee presentó en TED 2009 (Technology Entertainment and Design) una conferencia, en la que redefinió los principios de Linked Open Data presentándolos como tres reglas que se resumen en: a) asignar a todas las cosas conceptuales nombres que comienzan con http; b) obtener información importante de retorno a partir de los nombres; y c) la información obtenida debe contener relaciones. Se hace énfasis en esta conferencia en publicar los datos lo más pronto posible (Berners-Lee, 2009).

A partir de 2009 se ha producido un avance más rápido en el desarrollo de tecnologías de Linked Data. Se han liberado y usado algunas especificaciones de la W3C tales como: SPARQL, GRDDL, RDFa, VoID, se ha formado la comunidad del proyecto Linking Open Data y cada vez se observa un creciente uso en gobierno electrónico con numerosos catálogos de datos publicados y aplicaciones con funcionalidades específicas orientadas a dominios que combinan datos de varias fuentes de Linked Data (Berners-Lee, 2009; Sheridan et ál., 2010, Cyrille et ál., 2010).

En los últimos años se han desarrollado numerosas herramientas de apoyo a componentes arquitecturales de aplicaciones con Linked Data y se han propuesto patrones de publicación y consumo que serán tratados en las siguientes secciones. 
Por otra parte, existen iniciativas de apoyo a la investigación de Linked Data mediante la elaboración de proyectos y la conformación de asociaciones y redes tales como la Asociación Española de Linked Data AELID (AELID, 2010), la red iberoamericana de Linked Data, la red temática española de Linked Data, la red asociada al proyecto LOD2 entre otras (Heath,2011).

Paralelamente, las iniciativas de reutilización de información del sector público han tomado fuerza con algunas directivas publicadas en varios países como España, Inglaterra y Estados Unidos abriendo nuevas oportunidades para aplicación de tecnologías de Linked Data (Ramos, 2003; Abella, 2011).

\section{Tecnologías relacionadas con Linked Data}

Varias tecnologías están ya disponibles para publicación y consumo de Linked Data que pueden ser usadas también para integración y mejor gestión de datos corporativos. En este apartado se clasifican atendiendo a su finalidad: técnicas y herramientas que sirvan para la publicación, o técnicas y herramientas destinadas a facilitar el consumo de datos enlazados. Además se consideran en el apartado 3.3 algunos proyectos donde se utiliza Linked Data.

\subsection{Publicación}

La publicación en la Web semántica se puede realizar usando una variedad de herramientas y patrones de publicación siguiendo los principios de Linked Data, lo cual permite la interoperabilidad de los datos y reutilización en la Web. Sin embargo esto no implica el abandono de los sistemas existentes, si no más bien la adición de capas extras para conectar los datos en la Web. Estas capas pueden estar formadas 'por extractores de entidades para armar archivos RDF, almacenes de datos RDF, interfaces a Linked Data como Pubby, vistas RDF sobre Bases de Datos relacionales, APIs, uso de Sistemas de Administración de Contenidos (CMS) con RDFa o con salidas RDF, entre otros (Heath et ál., 2011). Adicionalmente la publicación de un conjunto de datos implica: escoger el conjunto de datos, aplicar una licencia abierta, publicar los datos, difundir la publicación, y evaluar su utilidad.

Para la descripción de Linked Data se usa el estándar de facto VoID (Vocabulary of Interlinked Datasets) el mismo que utiliza un esquema basado en RDF. Con VolD la localización y uso de conjuntos de datos enlazados se realiza de manera más efectiva (Cyganiak et ál., 2009).

\subsubsection{Herramientas para publicación}

Para publicar Linked Data se han desarrollado varias herramientas entre las cuales tenemos:

- D2R server es una herramienta para publicar bases de datos relacionales como Linked Data transformando el contenido de una base de datos relacional a RDF (Cyganiak et ál., 2009).

- Triplyfy es un plugin para aplicaciones web que revela las estructuras semánticas codificadas en bases de datos relacionales haciendo el contenido de bases de datos disponibles como RDF, JSON o Linked Data (Jaenicke, 2009).

- La Plataforma Talis proporciona hospedaje para contenido y datos RDF tanto para publicar datos como para desarrollo. Además ofrece interfaces de consulta y manipulación de datos con Kasabi, una plataforma estructurada de SaaS (Software as a Service) (Bizer et ál., 2008).

- Pubby es una interfaz para Linked Data que permite interactuar con puntos SPARQL (SPARQL endpoints) mediante consultas SPARQL (Cyganiak et ál., 2011).

- Paget es un marco de trabajo (framework) para construir aplicaciones con Linked Data (García et ál., 2011).

- Linked Media Framework: Es un servidor con actualizaciones y búsquedas semánticas (García et ál., 2011).

- Open Link Virtuoso Universal Server: Es un sistema de administración de bases de datos orientado a objetos que permite almacenar datos RDF y presentarlos con la funcionalidad de un punto SPARQL. Los datos en RDF pueden ser almacenados directamente en Virtuoso o creados a partir de bases de datos relacionales no RDF (Erling, 2010).

- Virtuoso Sponger: Permite transformar datos no RDF en RDF. Acepta como entradas páginas web $(X) H T M L$, páginas web $(X) H T M L$ con microformatos y servicios web como aquellos de Google, Del.icio.us, Flickr etc. Y crea RDF como salidas ( Bizer et ál., 2007).

- Sesame: Es un sistema formado por un repositorio, una máquina de búsqueda y un módulo de administración para añadir y borrar datos RDF e información de esquema. Sesame está siendo usado en algunas grandes compañías y agencias de gobierno para integración de datos (Broekstra et ál, 2004). 
- Jena TDB: Es un repositorio de datos RDF que permite añadir datos usando SPARQL/Update y permite consultas de datos usando el lenguaje SPARQL (Heath, T. et ál., 2011).

- 3Store: Es un repositorio de ternas RDF basado en MySQL. El software no presenta interfaces directamente al usuario pero puede ser consultado por varios servicios incluyendo el navegador Direct RDF (Aduna, 2009).

Respecto a los editores de Linked Data y validadores, cabe destacar:

- Hyena: Editor RDF, una mezcla de una wiki y una base de datos, disponible como aplicación de escritorio y como aplicación web (Rauschmayer, 2010).

- Vapour: Validador de Linked Data (García et ál., 2011).

- DrifftR: Editor y navegador de Linked Data (Nowack, 2007).

Existen otras herramientas, pero aquí se han seleccionado las que se han considerado más representativas, bien por su estado de avance, o por su grado de utilización de las tecnologías asociadas a Linked Data. Un listado, que se viene actualizando con regularidad, se puede encontrar en la wiki sobre herramientas de Web semántica, publicada y mantenida por el consorcio W3C (World Wide Web Consortium, 2010).

\subsubsection{Migración de datos}

Para facilitar la publicación de datos enlazados se puede migrarlos a RDF desde bases de datos relacionales, hojas electrónicas, XML, sitios web existentes tras ser indexados y puestos a disposición para consultas vía un servicio SPARQL. Los datos de salida pueden obtenerse en formatos como XML, RDF. Cada conjunto de datos debe tener sus metadatos y se los debe catalogar para facilitar las búsquedas (BernersLee, 2009).

Como ejemplo se puede citar el proyecto $D B p e-$ dia que usa scripts de PHP para extraer datos estructurados desde páginas de Wikipedia. Estos datos son convertidos a RDF y almacenados en el repositorio OpenLink Virtuoso el mismo que proporciona un punto de acceso SPARQL (Heath, 2011). Para acceder a los datos se usa Pubby.

\subsection{Consumo de Linked Data}

Los datos publicados en la Web de acuerdo a los principios de Linked Data forman parte de un espacio global de datos. En general en estos casos las aplicaciones explotan las propiedades de Linked Data entre las que podemos citar (Bizer et ál., 2007):

a) Representación y acceso estandarizado de datos que poseen descripciones.

b) Arquitectura abierta que permite descubrir nuevos datos en tiempo de ejecución a medida que están disponibles en la Web semántica.

Las tecnologías de Web semántica soportan un flujo de información punto a punto. Para requerimientos de integración, consultas e inferencias son útiles RDF, RDFa, OWL, SPARQL (Harris et ál., 2008).

El consumo de datos se puede realizar mediante aplicaciones generales o de dominio específico o mediante Linked Data mashups, aplicaciones que usan y combinan datos, presentaciones y funcionalidad procedentes de una o más fuentes. Los patrones arquitecturales de una aplicación de Linked Data pueden usar navegadores específicos rastreadores, o patrones de consulta de varias fuentes.

\subsubsection{Herramientas para consumo de Linked Data}

Los navegadores específicos (Linked Data browsers) permiten al usuario desplazarse entre diferentes fuentes de datos siguiendo los enlaces RDF. Por otra parte los documentos de la Web semántica pueden ser rastreados automáticamente siguiendo los enlaces RDF y los datos así obtenidos pueden ser sometidos a más sofisticadas capacidades de búsqueda (Heath et ál., 2011).

Entre los navegadores de datos RDF tenemos Object Viewer, Marbles, Disco, Tabulator, OpenLink RDF Browser, Zitgist Data Viewer, Dipper, Razorbase, iLOD, Fenfire y Quick \& Dirty RDF Browser. En cuanto a rastreadores RDF se tiene SWSE, Swoogle, URIBurner, los mismos que proporcionan descripciones RDF de recursos web en una variedad de formatos (Hartig et ál., 2010).

Existen máquinas de búsqueda de Linked Data, tales como Sig.ma, Falcons, SWSE, VisiNav, SWoogle y Watson, que proporcionan servicios de búsqueda, por palabras clave orientados a los usuarios. Adicionalmente, proporcionan enlaces a los documentos fuente que tienen las palabras clave mencionadas, lo cual ofrece mejores capacidades de interacción para explo- 
tar la estructura de los datos. Por ejemplo, se pueden filtrar resultados por clase y limitar los resultados a una subclase específica u obtener una lista de resultados con enlaces a entidades relacionadas (Hartig et ál., 2010).

Por otra parte se han desarrollado servicios sobre datos enlazados distribuidos como Sindi$c e$, que proporciona una API para que las aplicaciones puedan encontrar documentos RDF en la Web que se refieran a una cierta URI o contengan ciertas palabras clave. Estas palabras clave sirven para caracterizar las instancias de determinadas entidades de datos. Esto permite acceder a documentos de la Web semántica que contienen ciertas instancias de datos (Hausenblas, 2009). Otro ejemplo que brinda la posibilidad de reutilización de datos es Freebase, una base de conocimientos creada de forma colaborativa que ofrece también una API y un punto RDF para acceso en lectura y escritura a la información almacenada. Kasabi es otra aplicación, que ofrece la posibilidad de publicar datos enlazados y de consultarlos usando APIs predefinidos o elaborados por el usuario de acuerdo a sus necesidades permitiendo la generación de nuevos modelos de negocios (Zaino, 2011).

También se han desarrollado algunas herramientas para apoyar el consumo y fusión de fuentes de Linked Data. Es el caso de ALOE (Asisted Linked Data Consumption Engine) (AxelNgonga, 2011), que permite obtener información proveniente de varios servidores en la web como tripletas N3. Posee una demo para probar su potencialidad y es de fácil uso. Se usa para los ejemplos el repositorio DR2 Server Publishing (Cyganiak et ál., 2011). Otras áreas de desarrollo en apoyo a consumo son la definición de vocabularios como VolD,

\subsection{Proyectos con Linked Data}

Se han desarrollado varios proyectos usando las tecnologías mencionadas. El proyecto SWEO (2010) ha implementado un sitio web donde colecciona información acerca de aplicaciones que corren sobre Linked Data.

Por otra parte, el proyecto Linked Open Data presenta un diagrama actualizado periódicamente, de los conjuntos de datos publicados en formato Linked Data por la comunidad del proyecto y otros individuos y organizaciones. En este diagrama se puede observar una diversidad de conjuntos de datos clasificados por dominios (Cyganiak et ál., 2010). Más información acerca de estos conjuntos de datos se pueden obtener en el catálogo mantenido por CKAN (Comprehensive Knowledge Archive Network)
(Cyganiak, 2011). Para julio del 2011 se observan 1963 conjuntos de datos registrados en áreas tales como bibliotecas, ciencias de la vida, geografía, medios de comunicación y conjuntos de datos multi dominio como la DBPEDIA.

Como ejemplos de proyecto podemos citar Linked GeoData, que permite acceder a datos de localizaciones geográficas en OSM (OpenStreetMap), como RDF con interconexiones programadas con DBpedia y GeoNames (Riechert, 2011). El proyecto usa como repositorio de datos OpenLink's Virtuoso y el navegador Linked Geodata Browser.

Otra aplicación Linked Data es Talis Aspire, que ayuda a educadores a crear y administrar listas de recursos de aprendizaje, por ejemplo, libros, artículos de revistas, páginas web. La aplicación está escrita en PHP, soportada por la Plataforma Talis para almacenar, administrar y acceder a Linked Data. Es usada por miles de estudiantes de varias universidades. Los estudiantes y educadores interactúan con la aplicación mediante una interfaz web convencional mientras los datos que crean son almacenados en RDF (Clarke, 2009). En España el proyecto Morfeo promueve el desarrollo de software de fuentes abiertas, con estándares abiertos con un capítulo para el desarrollo d soluciones tecnológicas en el ámbito de Linked Data y Open Data. Otros proyectos que impulsan el uso de Linked Data son Aporta, Latac, Bi2RDF, Allen Brain Atlas, ePSI y Etalab (Abella, 2011).

En el campo de bibliotecas hay numerosos conjuntos de datos publicados como Linked Data, un listado de ellos se encuentra disponible en el informe del grupo incubadora del W3C, entre ellos tenemos: difusión de publicaciones científicas en Internet, lista de lecturas recomendadas de la universidad de Sussex, datos bibliográficos de Sudoc, bibliografía nacional británica, bibliografía nacional alemana, metadatos de tesis francesas, etc. (Isaac, 2011).

\section{Linked Data y administración electrónica}

Las agencias gubernamentales en algunos países publican cada vez más datos en Internet. Compartir estos datos permite más transparencia y alienta el uso público y comercial de información de gobierno. Las publicaciones se han realizado en varios formatos tales como PDF, HTML, CVS y como datos enlazados que son motivo de estudio en este trabajo. Algunos gobiernos han creado catálogos o portales tales 
como data.gov, data.gov.uk, data.gov.au, data.govt.nz, etc., para facilitar al público la localización y uso de los datos (Bennett et ál., 2009).

En España, gobiernos autonómicos como los de Euskadi, Cataluña o Asturias, corporaciones locales como los Ayuntamientos de Zaragoza o Gijón, y agencias estatales como el Instituto Geográfico Nacional, están comenzando a publicar sus datos de forma libre y gratuita (Villazón-Terrazas, 2011).

Según Abella (2011), España puede considerarse el segundo país más desarrollado en Open Data público de la Unión Europea y esta iniciativa podría crear 45000 empleos en 10 años. Con Open Data las Administraciones Públicas ponen a disposición de los ciudadanos los datos en su poder y permiten su combinación con datos privados para la creación de nuevos servicios o la mejora de los existentes. El estudio de Abella presenta varios proyectos e instituciones que están usando tecnologías de Linked Data promoviendo la reutilización de información del sector público.

En cuanto a demandas de aplicaciones para gobierno electrónico un estudio realizado por Nagy (2011), mediante un cuestionario en línea a ciudadanos, políticos, industriales, medios de comunicación, ciencia, revela que los potenciales usuarios de Linked Data buscan, entre otros, notas científicas, noticias de datos abiertos de administración pública, casos de uso e historias exitosas. El formato de datos preferido es RDF/XML. Ramos (2003), en su publicación sobre la reutilización de información del sector público reporta requerimientos de datos públicos jurídicos, información económica y financiera de las empresas, servicios de información meteorológica, información geográfica, científica, cultural, política, agrícola y pesquera. Los potenciales usuarios prefieren tener acceso a los conjuntos de datos fuente, tener información de versiones de los datos, alertas sobre datos regionales, y poder explorar y agrupar los datos.

\subsection{Estándares y ciclo de vida}

Para coordinar el trabajo en el desarrollo de estándares de gobierno electrónico y la Web semántica, la W3C ha formado el grupo eGov (eGovernment Interest Group) (World Wide Web Consortium, 2012), varios subgrupos sobre Web semántica, RDF, SPARQL, OWL, el Grupo GLD (Government Linked Data) (World Wide Web Consortium, 2010), el W3C PIG (Provenance Incubator Group) (World Wide Web Consortium, 2011) entre otros (Berners-Lee et ál., 2009).
Para ayudar en la publicación de datos en la Web el grupo de trabajo eGov de la W3C ha desarrollado algunas guías que enfatizan el uso de estándares y metodologías (Bennett et ál., 2009). Entre las recomendaciones emitidas por este grupo tenemos: a) publicar todos los datos disponibles, como leyes, regulaciones, documentos públicos, misiones, visiones, valores y metas en su formato de origen y en forma estructurada; b) crear un catálogo en línea bien documentado de los datos publicados y de sus versiones, incluyendo esquemas XML, DTDs y taxonomías que permitan la generación automática de diccionarios de datos proporcionando servicios de consulta, y explicar cómo encontrar los documentos en una colección, anunciar adiciones o cambios; y c) hacer que los datos sean legibles por humanos y máquinas enriqueciéndolos con semántica, metadatos e identificadores. Lo anterior implica crear nombres (URIs) para todos los recursos.

En el área de Linked Data, el grupo de trabajo GLD del consorcio W3C ha planificado estructurar recomendaciones para la construcción de ciclo de vida de proyectos de gobierno electrónico con Linked Data, definir pasos para crear y mantener conjuntos de datos gubernamentales usando principios de Linked Data, construcción de URIs, selección de vocabularios, versionado, estabilidad, organización de sitios web y posibles tecnologías a usar, entre otros.

Existen otras iniciativas de apoyo a la investigación en Linked Data en los diferentes países tales como la conformación de La Asociación Española de Linked Data AELID, que tiene como objetivo contribuir en la creación de un ecosistema de investigadores y emprendedores para favorecer el intercambio de conocimiento y experiencias (AELID, 2010).

Latif, et ál, (2009) proponen un ciclo de vida de Linked Data que puede aplicarse a proyectos de administración electrónica. Esta propuesta incluye la definición de Entidades tales como personas, empresas, asociaciones y entidades de investigación, actuando en diferentes roles para producir y consumir varios tipos de datos. En la Figura 1, se presenta el modelo de cadena de valor propuesto.

Otro modelo de ciclo de vida de Linked Data es el presentado por Hausenblas (2011), en el que se definen seis etapas: a) selección de datos, b) modelado, c) publicación, d) descubrimiento, e) integración, y f) definición de casos de uso. Para cada fase agrupa proyectos en marcha de apoyo.

En la discusión de la propuesta se sugiere considerar la naturaleza iterativa del ciclo de vida, y 
actividades de mantenimiento, control de versiones, autenticación y autorización entre otras.

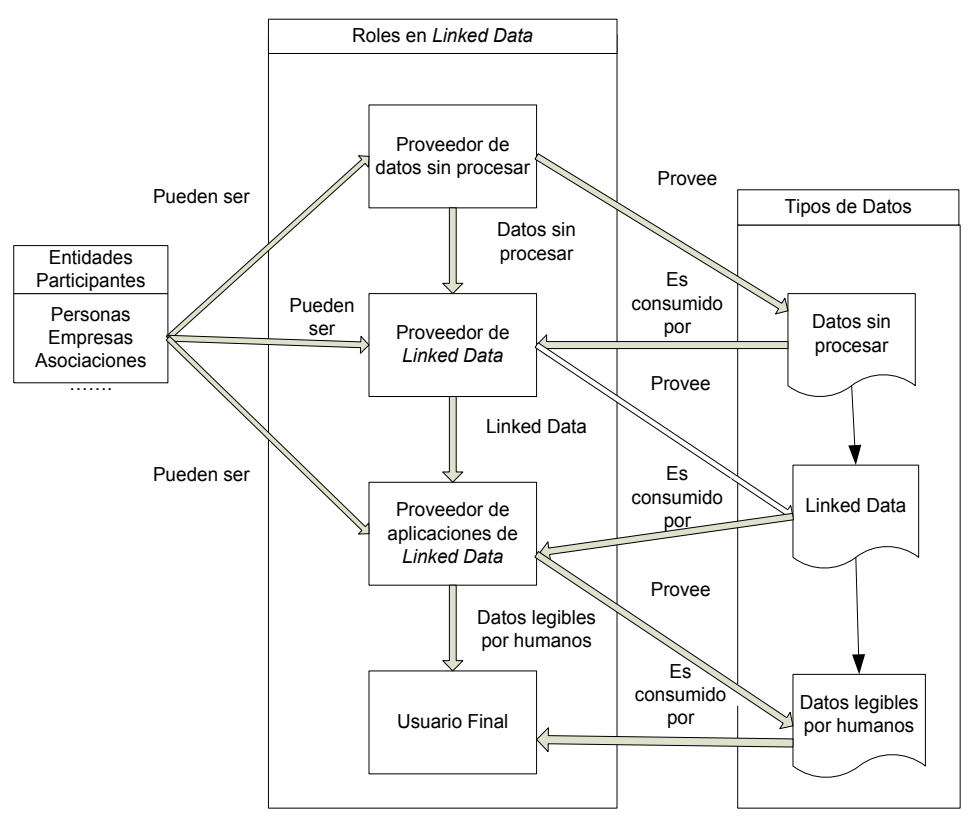

Figura 1. Cadena de Valor de Linked Data (adaptada a partir de Latif et ál, 2009).

En el proyecto LOD2 (Heath, 2011) se propone un ciclo de vida de Linked Data iterat ivo formado de las etapas: a) revisión manual y autoría de datos, b) enlaces y fusión, c) clasificación y enriquecimiento, d) análisis de calidad, e) evolución y reparación, f) búsqueda y exploración, g) extracción, y h) almacenaje y consulta.

\subsection{Aplicaciones de Linked Data}

a la administración electrónica

A partir del 2009, varios gobiernos alrededor del mundo comenzaronn a publicar datos en la Web, impulsados por el movimiento Open Access. Esto origina una gran oportunidad para probar el valor de estándares como RDF, OWL, SPARQL, GRDDL, SKOS (Simple Knowledge Organization System), etc. (Sheridan et ál., 2010).

La necesidad de integración de servicios para gobierno electrónico se puede satisfacer con las tecnologías ligadas a la Web semántica, por lo que varios sectores de gobierno, principalmente en Australia, Nueva Zelanda, UK, USA, España están produciendo Linked Data sobre estadísticas, registros de empresas, propietarios de tierras, registros de votantes, leyes, patentes, negocios, crímenes, educación, medioambiente, finanzas, investigación, transporte entre otros. Particularmente en data.gov.uk en Inglaterra y en data.gov en los Estados Unidos se publican significativos volúmenes de datos en RDF (Cagle, 2009; Sheridan, 2010). El proyecto Datagov Wiki (4) ha publicado varios billones de tripletas derivadas de datos publicados en Data.gov. En la implementación se usa Virtuoso Linked Data Server, SMILE Exhibit framework, el browser Graphite y Jquery (5). Actualmente los esfuerzos están dirigidos a capturar, propagar y mantener información de la procedencia de los datos enlazados o Linked Data.

En Inglaterra hay varias experiencias en este sentido, tales como el desarrollo de un vocabulario integrado del sector público con listas de servicios publicados como Linked Data en respuesta a una directiva de la Unión Europea (Berners-Lee et ál., 2006).

Por otra parte hay desarrollos para marcar recursos con metadatos semánticos para facilitar la recuperación de información, como la construcción y explotación de una ontología de metas de gobierno (acciones para alcanzar objetivos) y servicios, para promover la selección de un apropiado servicio para una meta en particular, y la elaboración de metadatos semánticos sobre varios puntos de vista de múltiples organizaciones o de una organización en múltiples instantes de tiempo (Harris et ál., 2008).

En Estados Unidos se desarrolla el proyecto educacional TWC LOGD (Tetherless World Constellation, s. d. b), con datos abiertos de gobierno usando tecnologías de Web semántica. Los grupos de datos de este sitio son convertidos a RDF desde varias de fuentes de datos tales como data.gov.

En el área legal se desarrolló el proyecto Caselex (Faro et ál., 2008), un servicio de información financiado por la Comisión Europea para proporcionar acceso a información legal que influye en varios países. El proyecto integra decisiones e interpretaciones de las cortes supremas de los estados miembros en un recurso multinacional combinado gracias al uso de metadatos semánticos.

En el ámbito legislativo en Inglaterra se lanzó en 2010 el sitio www.legislation.gov.uk. Este sitio concentra la legislación de toda Inglaterra en un solo lugar. Se presenta la legislación original y las versiones revisadas de Actos del Parlamento permitiendo la consulta de cambios de la legislación en el tiempo. El sitio proporciona una API que da completo acceso a los datos abiertos a desarrolladores y ciudadanos en general, demostrando como la Web de documentos y la Web semántica pueden ser combinadas. En este sitio se usa una base de datos nativa XML y una API (Reino Unido, s. d.) que soporta ne- 
gociación de contenido. Esta última característica hace posible direccionar diferentes versiones de un documento - -0 más generalmente de un recurso- a un mismo URI, de manera que los agentes usuarios puedan especificar qué versión requieren. Puede ser útil para publicar en diferentes formatos o idiomas (Sheridan, 2010).

En el caso de bibliotecas hay esfuerzos significativos para encadenar catálogos de bibliotecas permitiendo acceso a contenido de múltiples bibliotecas con información enlazada a otros archivos o bases de conocimientos. Hay ejemplos como la Biblioteca Nacional de España, la Biblioteca Americana del Congreso o la Biblioteca Nacional Alemana de Economía que publican sus títulos como Linked Data, o la Biblioteca Open Library que publica su catálogo en RDF. Otro caso es el sitio web DBLP (Digital Bibliography \& Library Project), que posee un enorme repositorio bibliográfico de artículos relacionados con ciencias de la computación. El sitio está administrado en la Universidad de Trier, Alemania (Ley, 2011). Otro esfuerzo en este sentido es el que se realiza en el proyecto Europeana financiado por la Comisión europea, con la participación de varios países, para la digitalización de colecciones regionales ofreciendo libre acceso en veintitrés idiomas.

Hay también aplicaciones que cubren las necesidades de comunidades específicas de usuarios como la DBpedia Mobile. Esta aplicación ayuda a turistas a explorar una ciudad basada en la posición GPS del dispositivo móvil y permite consultar y publicar información relacionada a los sitios que visita explorando una Web semántica geoespacial. DBpedia Mobile usa un visor de mapas y un browser de Linked Data basado en Fresnel (Becker et ál., 2009).

En los estudios de Alvite (2009), se preveía que el impacto de la Web semántica en los ámbitos jurídicos y de e-gobierno sería muy significativo. En 2011 ya se dispone de una mayor cantidad de datos de gobierno liberados en formato RDF tal como ocurre en el proyecto Data-gov Wiki (Tetherless World Constellation, 2009-201) y datos.gob.es, este último promoviendo la reutilización de datos públicos en España..

Actualmente los esfuerzos están dirigidos, entre otros, a capturar, propagar y mantener información de la procedencia de los datos.

Hay aún dificultades en la implementación de esta nueva tecnología a gran escala. En data.gov varios conjuntos de datos se convirtieron a RDF usando un algoritmo automático sin mayor empleo de curadores digitales que seleccionen información relevante, lo que limita el valor práctico de las tuplas RDF resultantes mientras que en data.gov.uk los datos fueron sometidos a un proceso de curación digital y son de alta calidad pero debido a los altos costos solo un grupo limitado de datos ha sido convertido en Linked Data (Cyganiak et ál., 2010).

Por otra parte, las iniciativas de reutilización de datos públicos abrieron nuevas oportunidades de utilización de las tecnologías de Linked Data, favoreciendo la creación de nuevos productos y servicios de valor añadido en el sector de los contenidos digitales y contribuyendo a la transparencia en la actuación de las administraciones públicas (Ramos, 2003). En el 2011 se emite un decreto en España sobre reutilización de información del sector público situándolo junto a países de vanguardia en materia de apertura de datos públicos, como Estados Unidos o Reino Unido. Además, la Comisión de la Unión Europea el 12 de diciembre del 2011 propone una nueva directiva tendiente a favorecer la reutilización de datos públicos para aplicaciones comerciales y no comerciales, revisando los lineamientos anteriores de cobros por acceso y uso de la información pública, y recomendando el uso de formatos adecuados para reutilización de la información

\section{Retos actuales}

En esta sección se propone una clasificación de los problemas relacionados con Linked Data por resolver y las posibles soluciones a los mismos.

\subsection{Diversidad de fuentes de datos}

Los datos a enlazar provienen de una diversidad de fuentes, con diferentes contextos sociales, lo cual hace difícil establecer enlaces adecuados entre los datos, de forma cooperativa, por la diferencia de significados. Por esto es importante poder grabar información del origen de los datos. Algunas herramientas como Tabulator ya lo permiten (Hartig et ál., 2010). Los usuarios requieren ir al origen de los datos, mirar metadatos de las fuentes de datos y asegurarse de tenerlos con licencia Creative Commons para reusarlos. El papel de los Data Curation Centres, que trabajan en definir y organizar adecuadamente los datos y metadatos y de los curadores digitales - usuarios "expertos" que seleccionan contenidos digitales que están disponibles en Internet sobre un tema y comparten su selección- es importante para facilitar la organización y acceso a datos con valor agregado.

Por otra parte, los datos fuente están publicados en diferentes formatos, muchos de ellos propietarios y según normativas variantes en el tiempo. Se debe enfatizar la importancia del uso de estándares de metadatos para facilitar la inte- 
gración de datos y analizar las mejores alternativas de versionado para conservar su historia. Por otra parte, sería conveniente disponer de herramientas para una selección y combinación automática de fuentes de datos.

\subsection{Colaboración para la publicación de datos}

Es urgente generar esfuerzos cooperativos en las diferentes tareas asociadas a la cadena de valor de Linked Data (ver fig 1), para optimizar tiempo y recursos. Con proyectos como Semantic Wiki, los administradores pueden colaborar en anotar y enlazar páginas. La colaboración en las administraciones en la creación de enlaces semánticos y en la construcción de ontologías permitiría reducir los costos de una actividad que consume recursos humanos especializados, abundantes y por consiguiente caros. Esta tarea puede ser facilitada con la creación de tutoriales y demostraciones de formas de aplicación de las tecnologías semánticas.

Por otra parte, es necesario definir estrategias para fortalecer relaciones entre diferentes unidades organizativas estatales -y con entidades privadas y académicas - para lograr un mejor desarrollo y difusión de soluciones.

En el caso de la ciencia la publicación abierta de los datos de estudios como Linked Data permite comprobar y enlazar resultados de experimentos, llegar a una mayor audiencia e incrementar la generación colaborativa de conocimiento.

La reticencia a entregar los datos por temores a pérdidas de control es un problema que se ha atenuado en algunos países con leyes que obligan a poner los datos abiertos, considerando que esto ayuda a identificar problemas y a solucionarlos. Para fortalecer esta iniciativa es necesario realizar estudios sobre los beneficios que obtienen los gobiernos y ciudadanos al publicar datos enlazados y difundir estos resultados.

La organización de grupos de trabajo colaborativos en temas específicos relacionados con Linked Data y la realización de congresos internacionales apoyan también al intercambio de información y su publicación.

\subsection{Herramientas y patrones de publicación}

La publicación de Linked Data se ha incrementado a partir de la publicación de las guías de diseño hecha por Berners-Lee en 2006 y su redefinición en 2009, en las que se recomienda publicar los datos lo más pronto posible siguiendo los principios de Linked Data. Al mismo tiempo se han desarrollado puntos de acceso
SPARQL, como el que se ofrece sobre los datos de DBpedia (OpenLink software, 2012).

En cuanto a añadir semántica en la Web actual, el consorcio W3C ha publicado una guía borrador para ayudar a decidir qué sintaxis y vocabularios usar para satisfacer las necesidades de editores y consumidores (Tension et ál, 2012). Las sintaxis de Microformatos, RDFa y Microdata permiten extraer datos de páginas HTML que pueden ser transformados en RDF. El proyecto Aporta también presenta una guía para facilitar la reutilización de datos públicos que debe ser considerada para publicación y consumo (España, Secretaría..., 2009.).

Además Google,Yahoo y Bing han optado por lanzar el uso de schema.org, un conjunto de esquemas que se pueden usar para marcar páginas web, impulsando el uso de Microdata, con licencias de uso, aunque anuncian que seguirán siendo compatibles con los vocabularios abiertos como los usados con RDFa (Franzon, 2012). Estas sintaxis se pueden implementar sin muchos cambios en las páginas web, de manera que una misma página se puede leer como página HTML o como página RDF.

Las propuestas indicadas son una alternativa a dedicar recursos para mantener páginas HTML e información en RDF/XML simultáneamente en cada sitio Web. Sin embargo, el uso de los enlaces estáticos causan un problema cuando desaparecen al no poder ser referenciados. Adicionalmente la información se dispersa en las páginas web y es más difícil su integración. Para evitar este problema, Berners_Lee (2008) sugiere que se añadan en los sitios Web opciones para publicar en RDF. De esta manera, los usuarios con navegadores especializados podrían usar dicha información.

Sin embargo, la experiencia de Inglaterra en la implementación del sitio Web data.gov.uk usando estándares Linked Data concluye que el mundo de Linked Data no está todavía listo para ser adoptado en gran escala a nivel gubernamental. Si bien los estándares son maduros y poderosos, se señala la necesidad de desarrollar patrones de publicación simples y prácticos para publicar los datos en RDF de manera que sean fácilmente adoptados, usados e implementados. Para facilitar el consumo, se han desarrollado APIs, pero aún se ve la necesidad de otro tipo de herramientas que faciliten las tareas de selección de información a enlazar, generación y mantenimiento de enlaces, control de calidad, etc. (Sheridan et ál., 2010).

Otro problema a considerar es que la interfaz para consulta de información en la Web actual la proporcionan en muchos casos los CMSs. Sin 
embargo, es necesario que se adapten a los nuevos requerimientos de la Web semántica de manera que permitan publicar y reutilizar contenidos en RDF o en un formato adecuado para el usuario. Algunos CMS, como Liferay y Drupal, disponen de soluciones que permiten publicar datos utilizando RDF, ofreciendo la posibilidad de incluir en un sitio Web datos externos mediante conectores a un Sparql Endpoint. A medida que se integren más funciones de la Web semántica en el núcleo de los CMSs, estos podrán usarse como frameworks para el desarrollo de interfaces entre datos de la Web semántica y contenidos de los sitios web que gestionen (Pastor-Sánchez, 2012).

Con respecto a facilitar la participación del usuario de Linked Data en actividades como el control de calidad de datos, sería adecuado que los browsers fueran también editores y la gente tuviera derecho a corregir información si detectan errores, verificando previamente dichas correciones.

Se requiere, adicionalmente, identificar maneras de incrementar la participación y acceso de los ciudadanos a los servicios ofrecidos por sistemas de gobierno electrónico, como, por ejemplo, sería buscar a qué fuentes acceden los ciudadanos para obtener sus datos.

\subsection{Problemas a superar sobre Linked Data en} relación con la administración electrónica

Hay algunos problemas que requieren urgente reflexión en relación con gobierno electrónico. Es necesario asegurarse que los datos públicos no violen leyes y regulaciones de los diferentes países. Se debe controlar la autenticidad de los datos y la privacidad de datos personales. Es necesario buscar mecanismos para incrementar la participación y acceso de los ciudadanos a los sitios que contienen Linked Data. García Marco (2009), analiza la convergencia de la Web semántica y la Web Social que ofrece una línea de investigación nueva. Por otro lado, se debe estimular a las agencias de gobierno para que publiquen datos usando las guías de diseño de Linked Data.

En resumen, las tecnologías de la Web semántica ofrecen un potencial para mejorar la interoperabilidad entre sitios y aplicaciones, la automatización en el descubrimiento de la información, opciones de búsqueda que son difíciles o imposibles sin información semántica. Además, el uso de ontologías permite la creación de conceptos más sofisticados y la inferencia de relaciones mejorando la calidad del contenido, y la integración de bases de datos. Sin embargo, se estima al 2011 una penetración del 1 al 5\% entre el público objetivo y se cataloga a la tecnología como en estado adolescente (Phifer, 2011).

\section{Conclusiones}

Las tecnologías asociadas a la web semántica y Linked Data son de gran utilidad y se están aplicando ya en la administración electrónica usando herramientas y estándares desarrollados para el efecto.

La estrategia de liberar datos a la mayor brevedad posible y paralelamente evolucionar hacia un modelo cercano a la web semántica es la escogida por algunas administraciones como Estados Unidos, Inglaterra o España. El hecho de poder construir capas semánticas sobre la web actual facilita el trabajo en esta dirección.

Debido al alto costo en recursos requeridos para implementar esta tecnología, se analizan alternativas colaborativas para la creación de catálogos de Linked Data mediante el apoyo de portales y herramientas sociales. Estas alternativas pueden contribuir al proceso de conversión de datos en Linked Data en actividades que pueden realizarse de manera colaborativa tales como anotar y enlazar páginas, seleccionar información relevante y realizar el control de calidad de los datos.

Por otra parte para acceder a Linked Data en la Web y navegar entre diferentes fuentes, se deben usar navegadores especializados, por lo que será necesaria una mayor difusión de los mismos o incrustarlos en los browsers de la web actual. Los navegadores web semánticos permiten acceder a diferentes fuentes de datos siguiendo enlaces autodescriptivos. Esto permite también a las máquinas de búsqueda de la web semántica seguir los enlaces para obtener datos enlazados en la web.

Otro problema que debe ser analizado es que los recursos de información provienen de diferentes fuentes que actualizan la información en el tiempo y que pueden proporcionar diferente información sobre un mismo recurso. Se deberían definir fuentes autorizadas sobre cada propiedad de un recurso, al igual que definir regulaciones de derechos sobre lo datos.

Para una implementación a gran escala es necesario desarrollar metodologías para generar y publicar Linked Data en la web, desarrollar algoritmos para interconectar datos automáticamente, desarrollar estándares para registrar información de las fuentes de datos, asegurar la privacidad, seguridad y preservación de los datos generados, crear herramientas para facilitar la búsqueda y despliegue de Linked Data. 
Otro problema a considerar es el uso de los enlaces estáticos que desaparecen continuamente, lo cual implica que aún el uso de RDFa no sea una solución para la publicación simultánea de páginas HTML y páginas RDF por la dificultad de seguimiento de los enlaces perdidos.

Las experiencias con Linked Data como la del Reino Unido han demostrado ser de mucha utilidad para encontrar información relacionada a un caso dado, a un tipo de dominio o información histórica. Sin embargo, aún no existen patrones de publicación y consumo simples para una aplicación a gran escala a nivel de gobierno y se continúan desarrollando alternativas de solución que faciliten la implementación de esta tecnología, como es el caso del desarrollo de APIs para facilitar el consumo de Linked Data.

\section{Referencias}

Abella, Alberto (2011). Reutilización de información Pública y Privada en España. http://www.navarra.es/NR/rdonly res/16750B44-0B82-4AB0-9DA7-17CDD0093216/18932 6/paper_reutilizacion_informacion_publica_privada_op.p df (2012-01-28).

Aduna (2009). OpenRdf.Org. http://www.openrdf.org/index. jsp (2011-08-5)

Alonso, José; Ambur Owen; et ál. (2009). Improving Access to Government through Better Use of the Web. http:// www.w3.org/TR/egov-improving (2011-07-5).

AELID (2010). Asociación Española de Linked Data. http://linkeddata.es/web/guest/home (2011-09-26).

Albornoz, Belén; Rivero, Martín (2007). Experiencias andinas de gobierno electrónico: La problemática de la participación ciudadana. http://www.flacso.org.ec/docs/gob_ electronico.pdf (2011-09-26).

Almarabeh, Tamara; Jordan, Amman. (2010). A General Framework for E-Government: Definition Maturity Challenges, Opportunities, and Success. // European Journal of Scientific Research. 39:1, 29-42.

Alvite, M.a Luisa (2009). Las bases de datos jurídicas y el uso del lenguaje XML en España. // Scire.15:1, 33-57.

Amutio, Miguel(2010). El Esquema Nacional de Interoperabilidad de España. http://www.peru.gob.pe/pm/portales/ portal ongei/eventos/Programas docu/43/Programa 30 8.pdf(2011-09-09).

AxelNgonga (2011). Assisted Linked Data Consumption, http://blog.aksw.org/2011/assisted-linked-data-consump tion (2011-08-22).

Becker, Christian; Bizer, Christian (2009). Exploring the geospacial semantic web with dbpedia mobile. // Journal of Web Semantics, 7:4, 278-286.

Bennett, Daniel; Harvey, Adam. (2009). Publishing Open Government Data.W3C Working Draft. http://www.w3.org/TR/2009/WD-gov-data-20090908/ (2011-07-02).

Berners-Lee, Tim (2006). Design Note: Linked Data. http://www.w3.org/Designlssues/LinkedData.html (201107-02).

Berners-Lee, Tim (2008). Sir Tim Berners-Lee Talks with Talis about the Semantic Web. Transcript of an interview recorded on 7 February 2008. http://talispodcasts.s3.amazonaws.com/twt20080207_TimBL.html (2011-09-16)
Berners-Lee, Tim (2009). Putting Government Data online. http://www.w3.org/Designlssues/GovData.html (201107-03).

Berners-Lee, Tim; Bizer, Chistian; et ál. (2009). Linked Data: The Story So Far. // Special Issue on Linked Data, International Journal on Semantic Web and Information Systems (IJSWIS). 5:3, 1-22.

Berners-Lee, Tim; Shadbold N.; et ál. (2006). The Semantic Web Revisited. // IEEE Intelligent System. 21:3, 96-101.

Berners-Lee, Tim (2009). Tim Berners-Lee on the next web. Transcript of an interview recorded on February 2009. // TED: ideas worth spreading. Feb. 2009. http://www.ted.com/talks/tim berners lee on the next web.html (2012-01-24).

Bizer, Chistian; Heath, Tom, et ál. (2008). Linked data on the web (LDOW2008). // Proceeding of the 17th international conference on World Wide Web. Beijing, China: ACM. 1265-1266

Bizer, Christian; Cyganiak, Richard, et ál. (2007). How to Publish Linked Data on the Web. // Computer and Information Science, 20:6, (2007) 43.

Broekstra,Jeen; Kampman, Ayohn (2004). RDF(S) Manipulation, Storage and Querying using Sesame. http://iswc2004.semanticweb.org/demos/03/index.html (2011-08-21).

Cagle, Kurt (2009). eGov Watch: The Importance of Data.Gov. http://broadcast.oreilly.com/2009/03/egov-watchthe-importance-of-d.html (2011-07-03).

Clarke, Chris (2009). A resource list management tool for undergraduate students based on linked open data principles. In Proceedings of the 6th European Semantic Web Conference, Heraklion, Greece, 2009: 697-707.

Codagnone, Cristiano; Wimmet, Maria (2007). Roadmapping eGovernment Research: Vision and Measures towards Innovative Government in 2020. Italy, My Print snc di Guerinoni Marco \& C., 2007.

COM (2003). El papel de la administración electrónica en el futuro de Europa. http://europa.eu/legislation_sum maries/internal_market/businesses/public_procurement/ I24226b_es.htm (2011-06-28).

CONATEL(2003). Programa nacional de gobierno electrónico y de la sociedad de la información. http://imaginar. org/index_archivos/gobierno/ecuador.pdf (2011-09-26).

Cyganiak, Richard (2011). The Data Hub. http://ckan.net/ (2011-07-02).

Cyganiak, Richard; Alexander, Keith; et ál. (2009). Describing Linked Datasets: On the Design and Usage of voiD, the "Vocabulary Of Interlinked Datasets". // Linked Data on the Web Workshop (LDOW 09). http://vmserver14. nuigalway.ie/xmlui/handle/10379/543 (2011-06-26).

Cyganiak, Richard; Bizer, Chistian (2011). Pubby. A Linked Data Frontend for SPARQL Endpoints. http://www4. wiwiss.fu-berlin.de/pubby/ (2011-07-02).

Cyganiak, Richard; Bizer, Christian (2009). D2R Server. http://www4.wiwiss.fu-berlin.de/bizer/d2r-server/ (201107-05).

Cyganiak, Richard; Jentzsch, Anja. (2010). The Linking Open Data cloud diagram LATC Project. http://richard.cyganiak.de/2007/10/lod/ (2011-06-25).

Cyganiak, Richard; Maali, Fadi; et ál. (2010). Self-service linked government data with dcat and gridworks. // Proceedings of the 6th International Conference on Semantic Systems. New York: ACM. 37, 1-3.

Cyrille, Axel; Ngomo, Ngonga; et ál. (2010). A time-efficient approach for large-scale link discovery on the web of data. http://svn.aksw.org/papers/2011/WWW_LIMES/public .pdf (2011-07-02). 
Ding, L.; et al. TWC LOGD: A Portal for Linked Open Government Data Ecosystems, Web Semantics: Science, Services and Agents on the World Wide Web (2011).

Erling, Orri (2010). Linked Data and Virtuoso. http://www. openlinksw.com/dataspace/oerling/weblog/Orri\%20Erlin g\%27s\%20Blog/1603 (2011-07-04).

España, Secretaría de Estado de Telecomunicaciones y para la Sociedad de la Información (2009). Guia aporta sobre reutilización de la información del sector público. Madrid: Secretaría de Estado de Telecomunicaciones y para la Sociedad de la Información. http://www.aporta.es/web/guest/descarga_manual2_apo rta (2012-01-29).

Faro, Sebastiano; Nannucci, Roberta (2008). TransEuropean access to national case law: The Caselex project. // Proceedings of the 2nd international conference on Theory and practice of electronic governance. J. Davies, J. Gibbons and S. Harris. New York, USA: ACM. 76-81.

Franzon, Eric (2011). Google, Yahoo! and Bing Announce Schema.org. http://semanticweb. Com/google-yahooand-bing-announce-schema-org_b20301 (2012-01-28).

Fuente, Carlos de la (2010). EI CTIC convierte a Asturias en sede mundial del 'open data'. http://www.clustertic.net/ headlines/stories/360-el-ctic-convierte-a-asturias-ensede-mundial-del-open-data (2011-07-02).

García Marco, Francisco Javier (2009). Perspectivas sobre el uso de la Web Semántica en el tratamiento de información y documentación legislativa. // Scire.15:1, 33-57

García, Roberto; Brunetti, Josep; et ál. (2011). Publishing and interacting with linked data. // Proceedings of the International Conference on Web Intelligence, Mining and Semantics. Norway: ACM. 1-12.

Gibbins, Nicholas; et ál. (2011). 3store. http://www.aktors.org/technologies/3store/ (2011-08-21).

Google (s. d.). Data-gov-wiki. http://code.google.com/p/datagov-wiki/(2011-08-25).

Harris, S.; Gibbons J.; et ál. (2008). Semantic technologies in electronic government. // Proceedings of the 2 nd international conference on Theory and practice of electronic governance. Cairo, Egypt: ACM. 45-51.

Hartig, Olaf; Langegger, Andreas (2010). A Database Perspective on Consuming Linked Data on the Web. http://www2.informatik.hu-berlin.de/ hartig/files/Hartig QueryingLD_DBSpektrum_Preprint.pdf (2011-07-04).

Hartig, Olaf; Sequeda, Juan; et ál. (2010). How to consume Linked Data on the Web. http://iswc2009.semanticweb. org/wiki/index.php/ISWC_2009_Tutorials/ How_to_Con sume_Linked_Data_on_the_Web (2011-07-05).

Hausenblas, Michael (2009). Linked Data Applications DERI Technical Report 2009-07-26. http://wtlab.um.ac.ir/ parameters/wtlab/filemanager/LD resources/other/lodapp-tr-2009-07-26_0.pdf (2011-06-20).

Hausenblas Michael (2011). Linked Data life cycles. http://linked-data-life-cycles. info/\#about (2012-01-25).

Heath, Tom; Bizer, Christian (2011). Linked Data: Evolving the Web into a Global Data Space, Morgan \& Claypool, 2011.

Hendler, James (2009). Web 3.0 Emerging. // Computer. 42:1 (2009) 111-113. http://lacnic.net/documentos/egov/ egov_argentina.pdf (2011-09-26).

Hendler, Jim; et ál. (2011). TWC LOGD: A Portal for Linked Open Government Data Ecosystems. http://tw.rpi.edu/ media/2011/07/13/d6fd/ twclogdaportalforlinkedopen.pdf (2012-01-28)

Isaac, Antoine,et ál.(2011), Grupo Incubadora de Datos Enlazados Bibliotecarios: Conjuntos de Datos, Vocabularios controlados y Conjuntos de Elementos de Metada- tos, vocabdataset/ (2012-02-02)

Jaenicke, Nadine (2009). Triplify. http://triplify.org/Overview (2011-07-06)

Latif, Atif; Hoefler Patrick; et ál. (2009). The Linked Data Value Chain: A Lightweight Model for Business Engineers. http://claudiawagner.info/publications/LOD-value chain.pdf (2011-07-06).

España. Ley 11/2007 de acceso electrónico de los ciudadanos a los Servicios Públicos. // Boletín Oficial del Estado. (22 de junio 2007).

Ley, Michael(2011). The DBLP Computer Science Bibliography. http://dblp.uni-trier.de/ (2011-07-02).

Linking Open Government Data. http://logd.tw.rpi.edu/tools technologies (2011-08-21).

Madrid, Lorenzo (2008). The economic impact of interoperability. Technical Report. http://osrin.net/docs/economic _impact_of_interoperability_v3.pdf (2011-06-15).

Montarcé, Pilar (2005). Programa Nacional de Gobierno electrónico, Argentina.

Nagy, Helmut (2011). The LOD2 Open Government Data Stakeholder Survey 2010. https://grips.punkt.at/display/ LOD2OGD/Introduction (2011-06-25).

Novamens (2011). Histourist. http://www.histourist.com/ home (2011-07-05).

Nowack, Benjamin (2007). DRIFTR. http://bnode.org/blog/ 2007/12/22/driftr-linked-data-browser-and-editor-screen cast (2011-07-02)

OpenLink software (2012). Virtuoso SPARQL Query Editor. http://DBpedia.org/sparql (2011-09-26).

Pastor-Sánchez, José-Antonio (2012). Los CMS como pieza fundamental en el despliegue de la Web semántica. // Anuario ThinkEPI. 6.

Phifer, Gene (2011). Hype Cycle for Web and User Interaction Technologies. http://www.planetlarg.net/my-scripts/ docs-to-read/gartner/hype_cycle_for_web_and_user_2 14448.pdf (2012-01-24)

Ramos, Simón (2003). La reutilización de la información del sector público: Aproximación al contenido de la propuesta de directiva 2002. // Revista General de Información y Documentación.13:2 (2003) 59-96.

Rauschmayer, Axel (2010). Hyena: Organize your ideas. http://proj.2ality.com/hyena/ (2011-07-02).

Reino Unido (s. d.). Legislation API. // data.gov.uk beta. http://data.gov.uk/dataset/legislation-api (2011-08-18).

Riechert, Thomas (2011). Linked GeoData. http://blog.aksw. org/category/projects/linked-geo-data (2011-08-21)

Salhofer, Peter; Tretter, Gerald, et ál. (2008). Goal-oriented service selection. Proceedings of the 2 nd international conference on Theory and practice of electronic governance. J. Davies, J. Gibbons and S. Harris. ACM. Cairo, Egypt: 60-66.

Sheridan, John (2010). Legislation.gov.uk. http://blog.law. cornell.edu/voxpop/tag/legal-linked-data/ (2011-05-16).

Sheridan, John; Tennison, Jeny (2010). Linking UK Government Data. Statistics. ACM Press. North Carolina: 1-

SWEO (2010). SWEO Community Project: Linking Open Data on the Semantic Web. http://www.w3.org/wiki/ SweolG/TaskForces/CommunityProjects/LinkingOpenDa ta/Applications (2011-06-27).

Tension, Jeni; et ál. (2012). HTML Data Guide.W3C Working Draft. http://www.w3.org/TR/2012/WD-html-data-guide20120112/ (2012-01-28).

Tetherless World Constellation (2009-2010). About the Datagov Wiki. http://data-gov.tw.rpi.edu/wiki/About_the_Datagov_Wiki (2011-08-20). 
Tetherless World Constellation (s. d. a). IOGDS: International Open Government Dataset Search. http://logd.tw. rpi.edu/tools_technologies (2011-08-21).

Tetherless World Constellation (s. d. b). TWC LOGD: http://code.google.com/p/data-gov-wiki/(2011-08-21).

World Wide Web Consortium (2010). Grupo Government Linked Data Charter. http://www.w3.org/egov/wiki/GLD Charter (2011-07-18).

World Wide Web Consortium (2010). Semantic Web Tools, http://www.w3.org/wiki/SemanticWebTools. (2011-0821).
World Wide Web Consortium (2011). W3C Provenance Incubator Group Wiki. http://www.w3.org/2005/Incubator /prov/ (2011-08-21).

World Wide Web Consortium (2012). eGovernment interest group. http://www.w3.org/egov/wiki/Main_Page (201107-20).

Zaino,Jennifer (2011). Kasabi Sees a Business Model In RDF Data. http://semanticweb.com/kasabi-sees-a-busi ness-model-in-rdf-data_b21385 (2011-05-16).

Enviado: 2011-11-11. Versión corregida: 2012-03-21. Aceptado: 2012-06-13. 
